\title{
The role of chromospheric mottles in the mass balance and heating of the solar atmosphere
}

\author{
G. Tsiropoula ${ }^{1}$ and K. Tziotziou ${ }^{2}$ \\ 1 National Observatory of Athens, Institute for Space Applications and Remote Sensing, Lofos Koufos, \\ 15236 Palea Penteli, Greece \\ e-mail: georgia@space.noa.gr \\ 2 Sterrekunding Instituut, Utrecht University, Postbus 80000, 3508 TA, The Netherlands \\ e-mail: K.Tziotziou@astro.uu.nl
}

Received 3 December 2003 / Accepted 12 May 2004

\begin{abstract}
High-resolution $\mathrm{H} \alpha$ observations of a solar region containing several mottles are analyzed. Mottles constitute the fine structure of the quiet solar chromosphere and are found at the boundaries of the network cells, where the magnetic field is mostly concentrated. The driving mechanism for mottles (as well as for spicules) is suggested to be magnetic flux cancellation which most likely involves magnetic reconnection. Magnetic reconnection explains the observed bi-directional flows and, furthermore, allows conversion of part of magnetic energy to heat. We estimate several physical parameters of mottles and report a detailed analysis of their temporal variations. We then consider their impact on the mass balance and the heating of the solar atmosphere. We find that less than $1 \%$ of the mass flux injected by these structures into the corona expands outward as solar wind. The major fraction of this flux returns back to the chromosphere and provides an explanation for the red-shifts observed in the transition region spectral lines. The energy released by magnetic dissipation is quantified in terms of different energy components. Using typical values for the parameters of these structures it is found that they can provide $\sim 20 \%$ to the energy budget of the solar corona, but only a small part of it goes to heating. This percentage, as well as the part of the energy that goes to heating, can be lower or higher depending on the relative frequency of these events and on their upward velocity. On the other hand, if one assumes that all the potential energy of the downflowing material is converted to heat the amount supplied to the chromosphere is really negligible.
\end{abstract}

Key words. Sun: chromosphere - methods: data analysis

\section{Introduction}

There have been numerous efforts to understand the energetics of the solar chromosphere and corona for several years. In recent years there is a general consensus that the key to solving this problem may be found in the understanding of the nature of the fine-scale transient events distributed all over the solar surface. Among these events are mottles which are the most prominent small-scale dynamic phenomena in chromospheric quiet regions. Mottles are rapidly changing hairlike jets of relatively cool material ejected from the low chromosphere and protruding into the hot corona with supersonic velocities of the order of $20-30 \mathrm{~km} \mathrm{~s}^{-1}$. A long-standing question is whether fine dark mottles are identified as spicules when observed at the limb. Although the majority of solar researchers believe that these two structures are somehow related, direct evidence does not exist for the time being due to observational reasons. However, in a previous work (Tsiropoula \& Schmieder 1997, hereafter Paper I) we derived several physical parameters of dark mottles (e.g. velocities, density populations, electron temperatures, electron densities etc.) and discussed their similarity to the corresponding spicule parameters. We suggested that this similarity together with similarities in dimensions and lifetimes provide indirect evidence to the identity of these structures. Two still very up to date comprehensive reviews of mottles and spicules properties can be found in Beckers (1972) and in the monograph of Bray \& Loughhead (1974), while for a recent review see Sterling (2000).

Fine mottles are usually organized in a complex geometric pattern over the solar disk. They cluster into small groups which are called chains and larger groups which are called rosettes. Chains and rosettes outline the boundaries of the chromospheric network where strong mixed polarity magnetic flux is mostly concentrated. It is now well established from investigations of high resolution magnetograms that new bipolar elements emerge continuously inside the cell interiors and are, subsequently, swept at the network boundaries by the supergranular flow (Wang et al. 1996; Schrijver et al. 1997). Interactions of the magnetic fields have as a result the enhancement of the flux concentration in the case of same polarities or its cancellation in the case of opposite polarities.

The bipolar nature of the flux cancellation process most likely involves magnetic reconnection which would drive material out of the reconnection region in opposite directions. 
Recently using data from the Multichannel Subtractive Double Pass (MSDP) spectrograph in conjunction with the cloud model we derived physical parameters of dark mottles and their temporal evolution (Tziotziou et al. 2003, hereafter Paper II). Another interesting characteristic of mottles is that they tend to occur at the same location several times. In Paper II we showed that intensity and velocity variations of mottles have a periodic behaviour with periods of the order of $5 \mathrm{~min}$. In a subsequent work (Tziotzou et al. 2004) by using a wavelet analysis we confirmed the existence of a predominant period of $\sim 5 \mathrm{~min}$ in dark mottles. From the temporal evolution of the line-ofsight velocity we found that the predominant motion is downward at their footpoints and alternatively upward and downward at their tops. These observations confirm earlier ones reported by Tsiropoula et al. (1993), who investigated a different set of data. Based on these observational findings we proposed a phenomenological model according to which mottles are due to magnetic reconnection. This process can occur not only at the network boundaries, but also inside the cells when neighboring pairs of flux tubes of opposite polarity are driven together by external plasma flows and reconnect. The suggestion that these structures are the products of magnetic reconnection is reasonable, since this process seems to be the most suitable physical mechanism that can explain the observed flow of material towards and away from the solar surface. However, for the time being, what drives magnetic reconnection in mottles is not clear.

Magnetic reconnection is probably the most suitable mechanism not only for releasing energy with possible implications for the heating of the solar chromosphere and corona (Priest \& Forbes 2000), but also for the transfer of cool gas from the chromosphere to the corona and the solar wind. Withbroe \& Noyes (1977) have estimated that energy fluxes of $5 \times 10^{6} \mathrm{erg} \mathrm{cm}^{-2} \mathrm{~s}^{-1}$ and $3 \times 10^{5} \mathrm{erg} \mathrm{cm}^{-2} \mathrm{~s}^{-1}$ are needed to maintain the quiet chromosphere and the quiet corona, respectively. It is worth noting at this point that although the chromosphere extends over a small height range and its temperature is of the order of $10^{4} \mathrm{~K}$, its radiative loss is ten times greater than that of the enormously extended hot corona. However, it is possible that the same mechanism that supplies energy to the chromosphere to sustain its heating rate supplies also energy that goes to heating the corona and accelerating the solar wind; e.g., there have been a number of suggestions in the past that spicules contribute substantially to both chromospheric and coronal energy fluxes. It is also suggested that spicules may play a major role in the mass balance of the solar outer atmosphere. A typical mass flux estimate needed to maintain the solar wind is $3 \times 10^{-11} \mathrm{~g} \mathrm{~cm}^{-2} \mathrm{~s}^{-1}$ (Ulmschneider 1971). It is reported that the globally averaged flux of hydrogen carried upwards by these structures is two orders of magnitude larger than the global solar wind mass flux; hence most of the material carried upward by spicules has undoubtedly to return again to the lower layers. Several observations of average redshifted EUV emission lines have indicated the presence of downflows in the transition region above the chromospheric network. These observations have led to the suggestion that the downflows represent spicular material falling back to the chromosphere (Pneuman \& Kopp 1978; Withbroe 1983). If mottles are indeed spicules seen on the disk then our results reported in Paper II offer the observational evidence to these suggestions.

Despite being a common feature of the solar chromosphere mottles are still a poorly understood phenomenon. Moreover, no quantitative study has been made so far regarding their impact in the mass balance and energy budget of the solar atmosphere by taking into account their detailed dynamics. The aim of this work is to derive several parameters of these structures, to present a systematic study of the variations of some of them and to obtain estimates of their role on the mass balance and energy budget of the solar atmosphere. For these estimates we will also use some spicule parameters when needed.

\section{Observations}

A time series of a $150^{\prime \prime} \times 120^{\prime \prime}$ solar region (see Fig. 1 of Paper II) containing 80 frames was obtained on May 14th, 2000. The observations were made in the $\mathrm{H} \alpha$ line with the MSDP spectrograph mounted on the French-Italian telescope THEMIS (Tenerife, Canary Islands) from 10:07 UT to 11:02 UT with a time cadence of $\sim 40.5 \mathrm{~s}$. Because of distortion due to seeing only the first 40 high quality images of the time series were used. The overall intensity pattern is almost preserved during the $\sim 27$ min of observations. After a closer inspection, however, individual mottles seem to change visibility during these minutes and, in particular, to oscillate about a "mean appearance" defined by the radial alignment, mean darkness and mean length. Furthermore, almost all mottles appear to rise again from the same source meaning that the mottle source may have several times the lifetime of a single mottle. The former property must be related to the small scale rearrangement of the magnetic field, while the latter to the large scale variations of the magnetic field.

\section{Analysis procedure}

Knowledge of the physical parameters of the various individual structures is crucial for their understanding and modelling, as well as for the solar atmosphere as a whole. Among the most widely applied methods for the deduction of different physical parameters of chromospheric structures using the $\mathrm{H} \alpha$ line is the contrast profile technique introduced by Beckers (1964). As it has already been pointed out by Alissandrakis et al. (1990), this method is appropriate for optically thin structures lying high enough above the chromospheric background and gives a good representation of the observed profiles and reliable values for four physical parameters: the source function $S$, the Doppler width $\Delta \lambda_{\mathrm{D}}$, the optical depth $\tau_{0}$ and the Doppler shift $\Delta \lambda_{\mathrm{I}}$ (see also Paper II, where we applied this method and derived these parameters for the current observations). After these four parameters are determined several others can be estimated, like the temperature $T_{\mathrm{e}}$, the number density in the second hydrogen level $N_{2}$, the electron density $N_{\mathrm{e}}$, the total particle density of hydrogen (i.e., neutral plus ionized) $N_{\mathrm{H}}$, the gas pressure $p$, the total column mass $M$, the mass density $\rho$ and the degree of hydrogen ionization $\chi$ (for details about the derivation of these parameters see Paper I). 

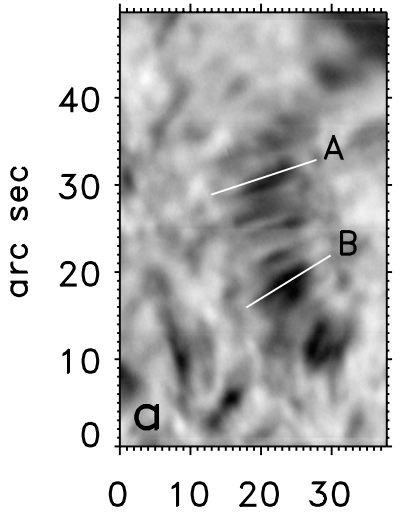
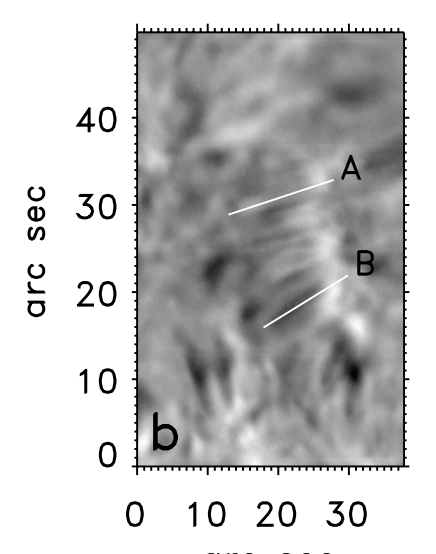

arc sec

Fig. 1. Images of a) intensity at $\mathrm{H} \alpha-0.48 \AA$ and b) Doppler velocity at $\mathrm{H} \alpha \pm 0.48 \AA$ (black corresponds to positive upward velocities) of the studied region observed with the MSDP on May 14th, 2000 at 10:15 UT. The overplotted lines, labelled "A" and "B", correspond to the cuts used for the time slice images of Fig. 3 (the feet of the mottles are at the right ends of the cuts).

\section{Results}

\subsection{General description of the parameters}

The procedure described in Sect. 3 was applied to the region shown in Fig. 1. The mean values and the standard deviations of the obtained parameters corresponding to the mottles of this region are given in Table 1. In the same table mean values of several other parameters of mottles used in this study are also given, i.e., the length $L$, width $l$, lifetime $t$. For two more properties needed in our calculations, e.g. the mean inclination of their axes $\theta$, and the mean axial velocity $v$ of the plasma along their axes, the results of Heristchi \& Mouradian (1992) derived for limb spicules were used. Thus for the mean inclination we take $\theta=29^{\circ}$. The axial velocity is usually studied by two different methods: the apparent upward motion and the Doppler shift of the spectral lines. According to these authors the first method gives an axial velocity distribution with a most probable value of $20 \mathrm{~km} \mathrm{~s}^{-1}$, while the second method gives a most probable velocity of $40 \mathrm{~km} \mathrm{~s}^{-1}$. In this study we use a mean value for the axial velocity $v$ equal to $25 \mathrm{~km} \mathrm{~s}^{-1}$ and examine the effect of different axial velocity values found in the range $20-40 \mathrm{~km} \mathrm{~s}^{-1}$, on the energetics of the mottles. It is obvious that the total number of mottles $N$ on the Sun plays also an important role on the energy budget of the solar atmosphere. According to Beckers (1963) the total number of mottles on the solar surface is estimated to be equal to $4 \times 10^{5}$. Later Beckers (1972) gives a value of $10^{6}$ for the total number of spicules on the solar surface. We can, of course, by counting the number of mottles in the field-of-view (FOV) of our observations estimate their total number on the solar surface. However, the number we find from this counting is rather low $\left(5 \times 10^{4}\right)$, probably because of the filament which covers a large part of the FOV. In this study we use a value of $4 \times 10^{5}$ for the total number of mottles and examine the effect that different values found in the range $5 \times 10^{4}-10^{6}$ would have in the energy budget of the solar atmosphere. The path length along the line-of-sight $d$ is equal to $2 \mathrm{Mm}$. For the estimation of the temperature and pressure

microturbulent velocities, $\xi_{\mathrm{t}}$, equal to $10 \mathrm{~km} \mathrm{~s}^{-1}$ and $15 \mathrm{~km} \mathrm{~s}^{-1}$ were assumed. Histograms of temperature (for $\xi_{\mathrm{t}}=15 \mathrm{~km} \mathrm{~s}^{-1}$ ), electron density $N_{\mathrm{e}}$ and mass density $\rho$, for all mottles found in all 40 frames of the region of Fig. 1, are given in Fig. 2.

Once the different physical parameters are obtained by the procedure described in Papers I, II and in the previous section their two dimensional maps for all 40 frames of the considered time sequence can be constructed. In order to examine the time evolution of the physical parameters along individual mottles, their values were averaged over a strip extending $0.3^{\prime \prime}$ on either side of the central axes of several mottles. It should be emphasized that following the mottle axis with time is a very delicate and difficult task. Good seeing conditions are required and careful coalignment of images taken at different times is necessary. Examples of the temporal evolution (time slice images) of the cloud velocity, temperature and mass density along the axes of two distinct mottles marked as A and B in Fig. 1 are given in Fig. 3. On the time slice images the contours of downward and upward velocities, as well as the contour of zero velocity have been overplotted. The velocity structures do not coincide with the limits of the intensity structures simply because the cloud model is not applicable at the ends of mottles. Furthermore, it is reasonable to infer that the maximal heights reached by spicular material are well above the apparent heights of spicules deduced from observations in visual limb images. Therefore the intensity cutoff need not be considered as the real upper limit; instead it would simply be the height at which $\mathrm{H} \alpha$ spicules become invisible. Temporal variations of all parameters show the quasi-periodic behavior described in Paper II with a period of the order of $5 \mathrm{~min}$.

Line-of-sight (LOS) velocity $\left(v_{\text {LOS }}\right)$ time slice images shown in Fig. 3 show the behaviour already described in Paper II. Mottles have a bi-directional flow structure in these maps showing predominantly downward motion at their footpoints and alternatively downward and upward at their tops. According to the model we proposed in Paper II the magnetic field drives the plasma both towards the corona and towards the photosphere, in the upper and lower part of the magnetic structure respectively. The bi-directional flows are followed by noticeable downflows which means that part of the material does not escape, but it returns to the solar surface. In the temperature time slice images it is interesting to notice that sometimes there is a temperature excess at the site where reconnection occurs (marked as "a" and "b" in Fig. 3), which could be a signature of local heating.

Figure 4 shows the variations of the LOS velocity, temperature, and mass density along the full extent of the mottles A and B (see Fig. 1) for two particular times when reconnection occurs, i.e. when downflows are observed at their lower parts and upflows at their upper parts. We consider positions where the velocity changes sign, as the reconnection site (at $\sim 5^{\prime \prime}$ for mottle A and at $\sim 9^{\prime \prime}$ for mottle B). The mottles have higher temperatures at the reconnection site. This is consistent with an energy supplied from the reconnection process. The temperature of the outflowing, as well as of the downflowing plasma, decreases. An explanation to this maybe that the outflowing and downflowing plasma is cooled down as a result of conduction and radiation losses along the reconnected field lines. 
Table 1. Physical parameters of dark mottles.

\begin{tabular}{lccc}
\hline \hline Parameter & $\begin{array}{c}\text { Average } \\
\text { value }\end{array}$ & $\begin{array}{c}\text { Standard } \\
\text { deviation }\end{array}$ & Comments \\
\hline$N_{2}, \mathrm{~cm}^{-3}$ & $4.2 \times 10^{4}$ & $2.0 \times 10^{4}$ & \\
$N_{\mathrm{H}}, \mathrm{cm}^{-3}$ & $9.9 \times 10^{10}$ & $2.5 \times 10^{10}$ & \\
$N_{\mathrm{e}}, \mathrm{cm}^{-3}$ & $6.4 \times 10^{10}$ & $1.6 \times 10^{10}$ & \\
$M, \mathrm{~g} \mathrm{~cm}^{-2}$ & $2.2 \times 10^{-5}$ & $5.7 \times 10^{-6}$ & \\
$\rho, \mathrm{g} \mathrm{cm}^{-3}$ & $2.2 \times 10^{-13}$ & $5.7 \times 10^{-14}$ & \\
$T, \mathrm{~K}$ & $1.8 \times 10^{4}$ & $2.9 \times 10^{3}$ & For $\xi_{\mathrm{t}}=10 \mathrm{~km} \mathrm{~s}^{-1}$ \\
$T, \mathrm{~K}$ & $1.0 \times 10^{4}$ & $2.9 \times 10^{3}$ & For $\xi_{\mathrm{t}}=15 \mathrm{~km} \mathrm{~s}^{-1}$ \\
$p, \mathrm{dyn} \mathrm{cm}{ }^{-2}$ & 0.42 & 0.1 & For $\xi_{\mathrm{t}}=10 \mathrm{~km} \mathrm{~s}^{-1}$ \\
$p, \mathrm{dyn} \mathrm{cm} \mathrm{cm}^{-2}$ & 0.24 & 0.1 & For $\xi_{\mathrm{t}}=15 \mathrm{~km} \mathrm{~s}^{-1}$ \\
$\chi$ & 0.65 & & \\
$L, \mathrm{Mm}$ & 10 & & \\
$l, \mathrm{Mm}$ & 1 & & \\
$t, \mathrm{~s}$ & 300 & & \\
$\theta,{ }^{\circ}$ & 29 & & and in the range $5 \times 10^{4}-10^{6}$ \\
$d, \mathrm{Mm}_{N}$ & 2 & & and in the range $20-40$ \\
$v, \mathrm{~km} \mathrm{~s}^{-1}$ & $4 \times 10^{5}$ & & \\
\hline
\end{tabular}
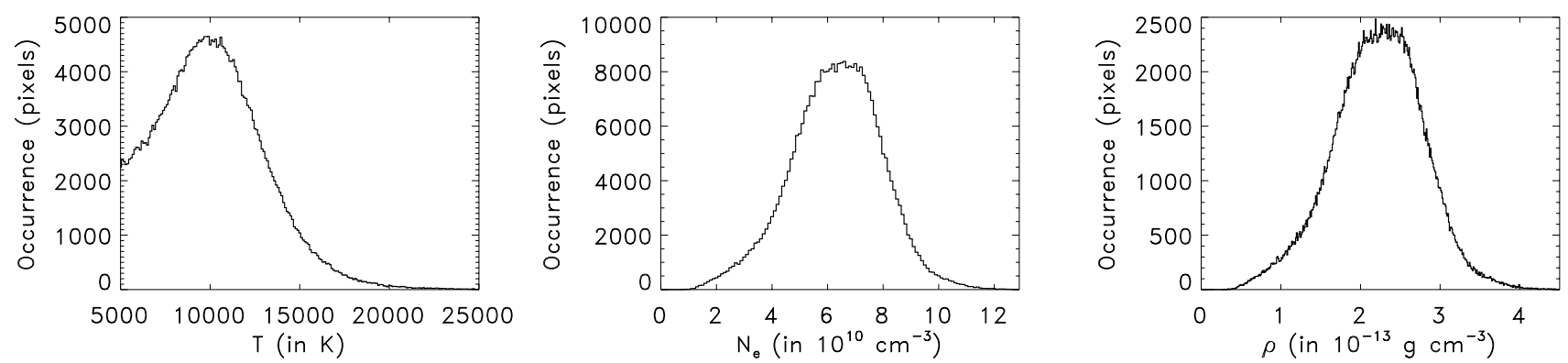

Fig. 2. Histograms of temperature $T$ (for $\xi_{\mathrm{t}}=15 \mathrm{~km} \mathrm{~s}^{-1}$ ), electron density $N_{\mathrm{e}}$ and mass density $\rho$ for the mottles found in the region shown in Fig. 1.

We suggest that the corona may contain surprisingly large volumes of very cool plasma, much of which is probably of chromospheric origin. It appears that only a fraction of this cool material is detectable optically in $\mathrm{H} \alpha$ because of the relatively low $n=3$ to $n=2$ population ratio, while its opacity is also likely to be low. It is not impossible, however, that the temperature decreases first and then increases from a height not visible in $\mathrm{H} \alpha$ (Matsuno \& Hirayama 1988). The increasing temperature with height may well be due to the heating by the penetrating radiation in the EUV emission of the transition region. The mass density decreases both from the reconnection site towards the footpoints and towards the top. Several other parameters (like e.g. the various population densities) have at the reconnection site their highest values consistent with the accumulation of mass from the reconnection. Moreover, all these parameters decrease downwards as well as upwards, but with a steeper gradient (see Fig. 4). Figure 5 shows the variations as functions of time of the velocity, intensity and temperature taken as the averaged values across a strip extending from $7.75^{\prime \prime}$ to $8.25^{\prime \prime}$ (see Fig. 3). The intensity of mottles during the downflow phase is lower than the intensity during the upflow+downflow phase. An explanation of this is evidently that the material cools and absorbs more in $\mathrm{H} \alpha$ when the gas falls back down. That the material is cooler during the downflowing phase can also be seen from the temperature variations in Fig. 5.

\subsection{The role of mottles in the mass balance and heating of the solar atmosphere}

\subsubsection{Mass balance}

In order to examine the role of mottles in the mass (and in the energy, see Sect. 4.2.2) balance of the solar atmosphere, we will take the mean values of the different parameters of Table 1 as representative ones. We also remind that mottles arise at the network boundaries or inside the cells as bursts of material attaining maximum upward or downward velocities $v$ of the order of $25 \mathrm{~km} \mathrm{~s}^{-1}$ and tend to occur several times at the same location with a typical duration of $\sim 5 \mathrm{~min}$.

The upward mass flux of mottles depends on: a) the fraction $f$ of the solar disk covered by these structures at any given moment; b) the mass density $\rho$; and c) the axial upward mottle velocity $v$. Taking the total number of mottles on the solar surface to be equal to $4 \times 10^{5}$ the fraction of the solar surface they cover is $\sim 0.05$. The mass density is taken from Table 1 $\left(\rho=2.2 \times 10^{-13} \mathrm{~g} \mathrm{~cm}^{-3}\right)$. As at any given moment only half 

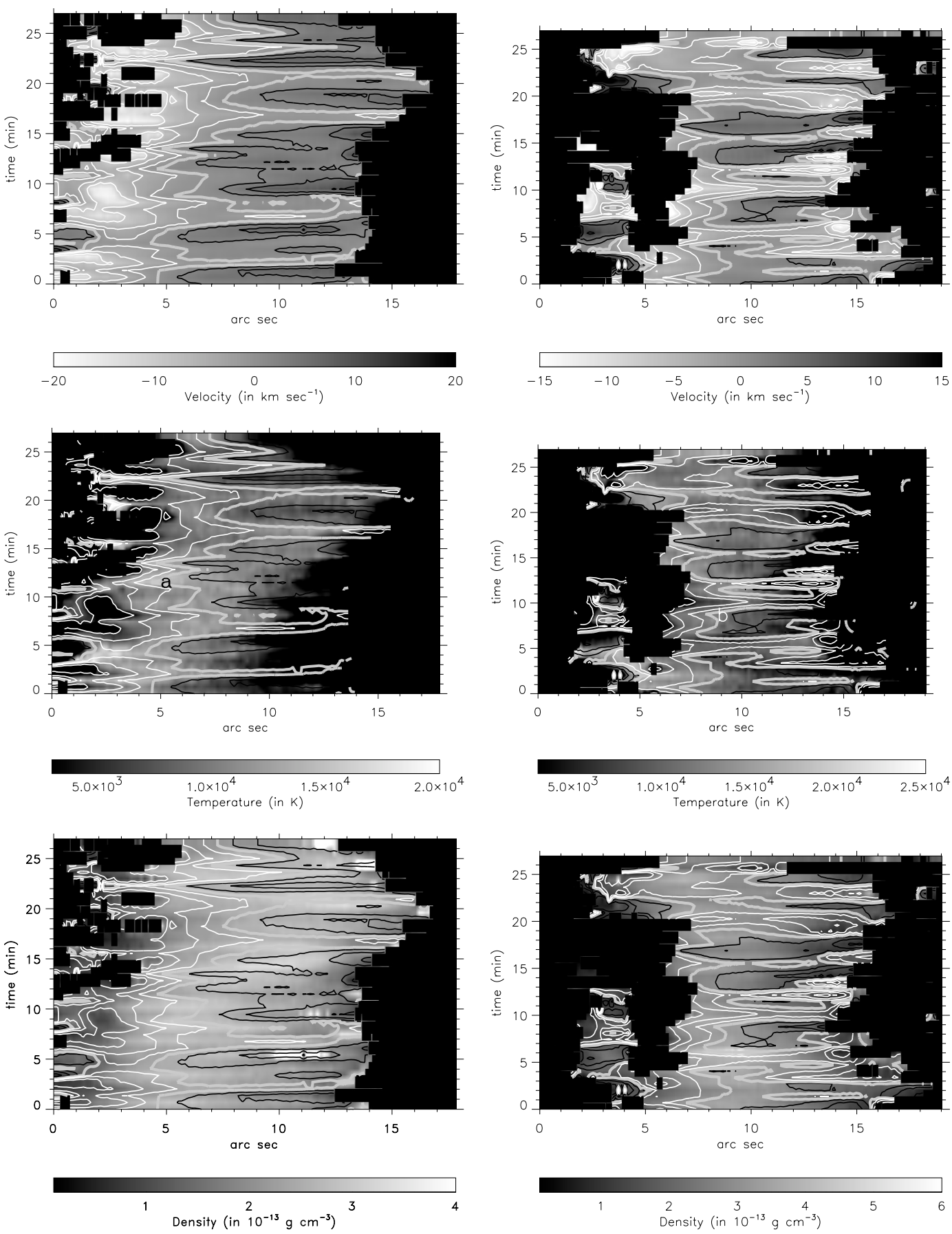

Fig. 3. Time slice images of the velocity $v_{\mathrm{LOS}}$ (top), temperature $T$ (middle) and mass density $\rho$ (bottom) from cuts along the central axes of the two mottles marked as A (left) and B (right) in Fig. 1. On all images, the black contours denote upward (positive) velocities, the white contours downward velocities while the thick gray line represents the zero velocity contour. Position " 0 "' "corresponds to the feet of the structures. On the temperature images, "a" and "b" mark the reconnection regions used for Fig. 4.

of the mottles are showing upward motion and from them only half of the material is flowing upwards (assuming that the reconnection occurs in the middle of the structures) the globally averaged mass flux carried upwards can be obtained from the relation

$F_{\mathrm{M}}=\frac{1}{2}\left(\frac{1}{2} f \rho v\right)$ and is equal to $7.1 \times 10^{-9} \mathrm{~g} \mathrm{~cm}^{-2} \mathrm{~s}^{-1}$. This value of the outward mass flux is two orders of magnitude larger than the outward flow of mass from the corona due to the solar wind which is equal to $3 \times 10^{-11} \mathrm{~g} \mathrm{~cm}^{-2} \mathrm{~s}^{-1}$ (Ulmschneider 1971). Thus it seems that these structures provide the means by which coronal mass losses in the solar wind can be compensated by mass ejected from below establishing the overall mass balance in the solar atmosphere. We can also conclude from the above estimate that less than $1 \%$ of the material of mottles escapes. 

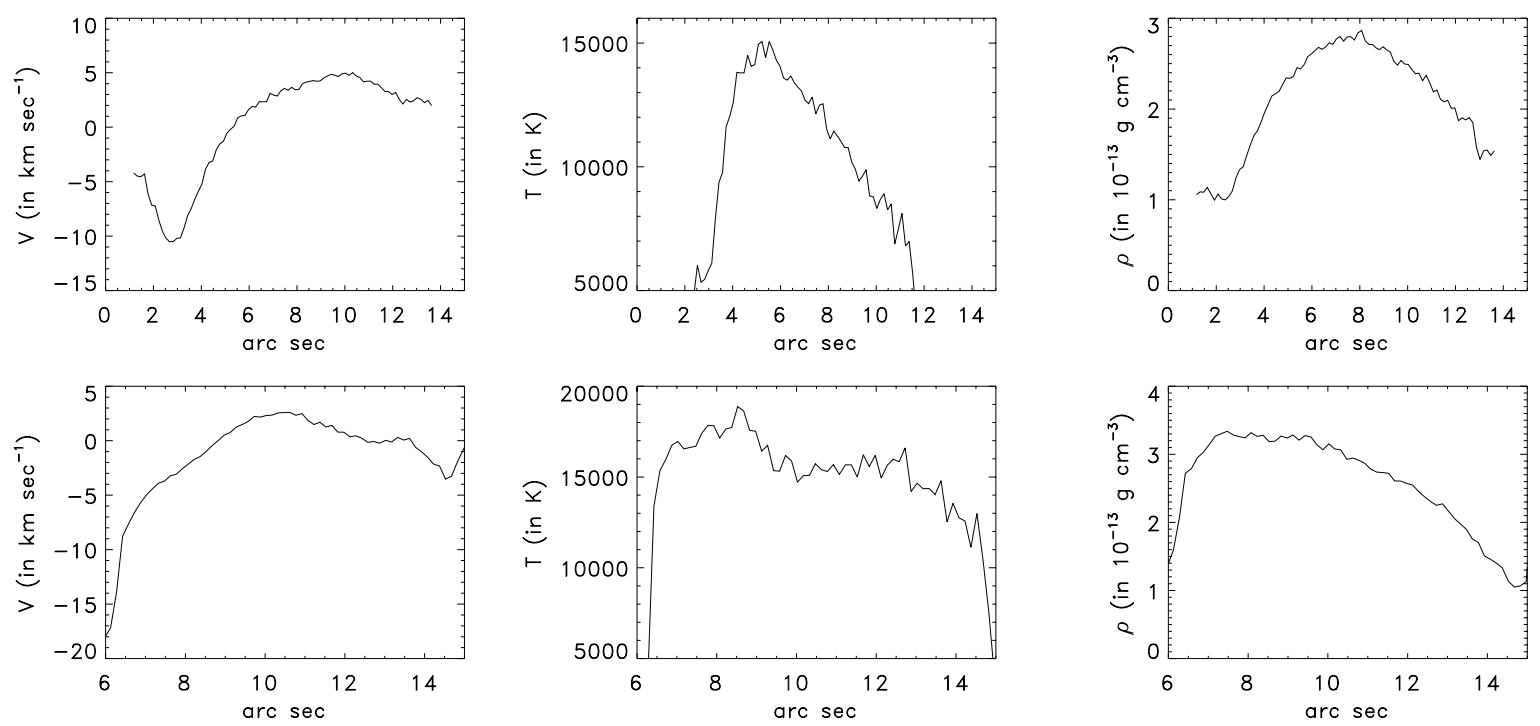

Fig. 4. Variation of the velocity $v_{\mathrm{LOS}}$, temperature $T$ and mass density $\rho$ along the central axes of the two mottles marked as A (top row) and B (bottom row) in Fig. 1 at the time of the reconnection marked as "a" and "b" respectively in Fig. 3 ("0" corresponds to the feet of the structure).
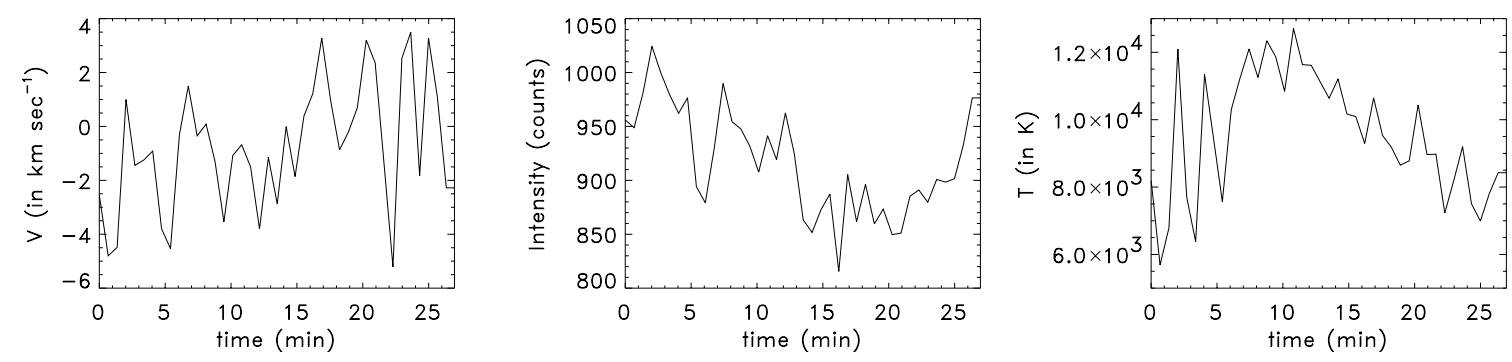

Fig. 5. Variation with time of the LOS velocity (left), intensity (center) and temperature (right) at one distinct position of the mottle marked as A in Fig. 1.

The remainder returns back to the chromosphere. A number of EUV observations have shown widespread downflow of matter at temperatures near $10^{5} \mathrm{~K}$ in the network regions bordering supergranules (Pneuman \& Kopp 1978; Withbroe 1983). Both from their spatial location and magnitude it was claimed that these downflows are related to the spicular material falling back. Our result regarding mottles provides one more indirect evidence that points to the identity of spicules and mottles. From the mass flux falling back $\left(\sim 0.99 F_{\mathrm{M}}\right.$ and the mass density at the transition region $\left(\rho_{\mathrm{tr}}=6.7 \times 10^{-15} \mathrm{~g} \mathrm{~cm}^{-3}\right)$ the downflow velocity due to the return of mottles' material can be estimated from the relation

$v_{\mathrm{f}}=\frac{F_{\mathrm{f}}}{\rho_{\mathrm{tr}}}$.

A value equal to $\sim 11 \mathrm{~km} \mathrm{~s}^{-1}$ is obtained. It is interesting to note that the value of the downflow velocity obtained from the above relation is consistent with the value attributed to the downflow velocities observed at the transition region (see, e.g., Hansteen et al. 2000). Observations in the EUV range seem to indicate that $\mathrm{H} \alpha$ spicules emit in EUV as well. It is suggested that these emissions possibly have their origin in a thin sheath which separates the cool $\mathrm{H} \alpha$ emitting spicular material from the hotter surrounding environment (Withbroe 1983).

\subsubsection{Energy budget}

As has been already noticed by Blake \& Sturrock (1985) the height attained by spicules is much greater than either the barometric scale height or the ballistic scale height. The barometric scale height is in the range $300-600 \mathrm{~km}$. The ballistic scale height $\left(v^{2} / 2 g\right)$ for a typical spicule velocity of $25 \mathrm{~km} \mathrm{~s}^{-1}$ is $1300 \mathrm{~km}$. Thus the material must be subject to an upward force which counteracts the effect of gravity.

Magnetic reconnection is considered to be an efficient means of converting magnetic energy into kinetic and thermal energy. We will give a rough estimate of the magnetic energy dissipated at the reconnection region which is given by

$F_{B}=\frac{B^{2}}{8 \pi} \frac{b}{2} V_{\mathrm{r}}$

where $B$ is the non-potential component of the magnetic field, $V_{\mathrm{r}}$ is the volume that contributes magnetic energy to the reconnection region and $b$ is the birthrate of mottles which is obtained from their total number seen on the disk and divided by their mean lifetime $(b=1333$ mottles/s for a total number of mottles equal to $4 \times 10^{5}$ ). We further suppose that half of them show the signature of reconnection each time and the other half are downflowing. The actual reconnection region itself is much smaller than this volume and is determined primarily by dissipative processes (Forbes \& Priest 1987). However, as has been 
noted by Cargill (1994), the reconnection volume does not determine the volume heated, since reconnection field lines are continually swept away from the reconnection site at the same time as unreconnected field lines are convected into it. The volume of the reconnection region is taken equal to $\pi\left(\frac{l}{2}\right)^{2} h$, where $l$ is the mean width of the mottles and $h$ is the length along the axis of a mottle along which reconnection is occurring. This length is taken equal to the vertical extension of the temperature excess region (see Fig. 3 second row, as well as Fig. 4 middle column), i.e. $\sim 3000 \mathrm{~km}$. The resulting volume is equal to $2.35 \times 10^{24} \mathrm{~cm}^{3}$. If the velocity of the upward moving plasma is equated to the local Alfvén speed given by

$v_{\mathrm{A}}=\frac{B}{\sqrt{4 \pi \rho}}$

then a knowledge of the plasma density leads to a value for the magnetic field strength. Thus from Eq. (4) a magnetic field strength of $\sim 4.1 \mathrm{G}$ is obtained. This is the classical SweetParker steady-state solution which assumes that the outflow pressure is the same as that at the neutral point.

Under the above assumptions we find from Eq. (3) that the magnetic energy dissipated at the reconnection region is of the order of $1.1 \times 10^{27} \mathrm{erg} \mathrm{s}^{-1}$ or $1.8 \times 10^{4} \mathrm{erg} \mathrm{cm}^{-2} \mathrm{~s}^{-1}$ when averaged over the surface of the Sun. This value is in itself too small to be significant in solar atmospheric heating.

Of course, in attempting to derive estimates of field strength and total energy release from the observations there are several important points to note. First of all, the volume of the reconnection region is largely unknown. Secondly, only a fraction of the field can reconnect, in particular the non-potential component and actually even a fraction of this component can reconnect, since the total helicity must be preserved before and after reconnection. Therefore, equating the observed velocities to the Alfvén speed gives a gross underestimate of the field strength. Furthermore, what we observe is only the bulk plasma flow, which is not the same as the small-scale reconnection jets at the diffusion region. The reconnection jets are expected to be at the Alfvén speed, but the bulk flow can be considerably smaller (Antiochos, private communication).

The estimate described above is based on the idea that the released energy is furnished by magnetic dissipation. The most effective and plausible mechanism for this dissipation is magnetic reconnection. We will try to give below a more complete description of the energetics taking into account the different energy components. We assume that the energy produced by magnetic dissipation at the reconnection site goes into thermal energy, kinetic energy of the bulk flow, gravitational energy, wave energy, as well as conduction and radiation losses. Our aim is to obtain order of magnitude estimates of the different processes that contribute to the energy budget and to derive an estimate of the energy released. We assume that the reconnection point is located in the middle of the structure. We also consider that the part of the structure below the reconnection point is pulled down and thus the energy provided by the dissipation of the magnetic energy goes to the heating of the chromosphere, while that dissipated above the reconnection point goes to the heating of the corona. Thus we can separate the energy fluxes into those travelling towards and those travelling away from the observer according to the upward $v_{+}$and downward $v_{-}$velocity directions (see also Winebarger et al. 1999). We, furthermore, consider two phases: a) the reconnection phase (upward+downward flow) and b) the downflow phase (see Paper II, Fig. 8). We, finally, consider the energy balance per event and then integrate over the solar surface.

\section{Reconnection phase Energy balance per event requires}

$F_{\text {mag } \pm}=F_{\text {rad } \pm}+F_{\text {cond } \pm}+F_{\mathrm{w} \pm}+F_{\mathrm{K} \pm}$

expressed in terms of fluxes, e.g. in erg $\mathrm{cm}^{-2} \mathrm{~s}^{-1}$, where "+" and "-" represent fluxes toward and away from the observer, respectively. The first term on the right hand side of Eq. (5) corresponds to the radiative flux, the second term to the conductive flux along the magnetic field lines, the third to the wave energy flux. Although waves must be present their precise modes and amplitudes are not known. Thus the third term of Eq. (5), although probably important, will simply be omitted from the following discussion. The last term of Eq. (5) is the sum of three terms

$F_{\mathrm{K} \pm}=F_{\text {kin } \pm}+F_{\text {enth } \pm} \pm F_{\mathrm{g} \pm}$

1. The first term of the right hand side of Eq. (6) represents the kinetic energy flux, $F_{\text {kin } \pm}=\frac{1}{2} \rho v_{ \pm}{ }^{3}$.

2. The second term is the enthalpy flux, $F_{\text {enth } \pm}=\frac{\gamma}{(\gamma-1)} p v_{ \pm}$.

3. The third term is the gravitational energy flux, $F_{\mathrm{g} \pm}=$ $\rho g \frac{L}{2} v_{ \pm}$.

where $\gamma$, is the ratio of the specific heats and $g$ is the acceleration due to solar gravity. $\gamma$ is taken equal to $\frac{5}{3}$, although it must be smaller than this value since we are dealing with a partially ionized gas. Assuming that the mean values for the upward and downward parameters are equal (e.g. the velocity $v_{ \pm}=25 \mathrm{~km} \mathrm{~s}^{-1}$, the mass density $\rho_{ \pm}=2.2 \times 10^{-13} \mathrm{~g} \mathrm{~cm}^{-3}$,

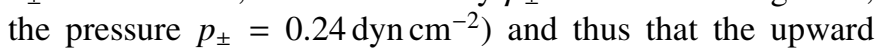
and downward fluxes are equal, then the total flux components per event during the reconnection phase are given by $F_{i, t}=F_{i,+}+F_{i,-}=2 F_{i, \pm}$. For the net radiative loss rate per unit volume we use the analytical expression given by Nagai (1980)

$e_{\mathrm{rad}}=\alpha(T) N_{\mathrm{e}}^{2} \chi g(T)$

given in $\operatorname{erg~cm}^{-3} \mathrm{~s}^{-1}$, where $g(T)$ is a semi-empirical function of the temperature, $\alpha$ is the radiative reduction coefficient and $\chi$ is the ionization degree taken equal to 0.65 (Table 1). According to Nagai (1980) for $T=10^{4} \mathrm{~K}$, $g(T) \sim 10^{-23} \mathrm{erg} \mathrm{cm}^{3} \mathrm{~s}^{-1}$ and $\alpha(T) \sim 0.1$. Using these values we find that the net radiative loss rate, $e_{\text {rad }}$ is equal to $2.7 \times 10^{-3} \mathrm{erg} \mathrm{cm}^{-3} \mathrm{~s}^{-1}$. To calculate the total radiative losses the radiative volume $V=\pi\left(\frac{l}{2}\right)^{2} L$ is required, where $l$ is the mean width of the mottles and $L$ their vertical extent. The radiative loss rate due to radiative cooling is then given by

$E_{\mathrm{rad}, \mathrm{t}}=e_{\mathrm{rad}} V$ 
and is of the order of $2.1 \times 10^{22} \mathrm{erg} \mathrm{s}^{-1}$, while the radiative flux given by

$F_{\text {rad,t }}=e_{\text {rad }} L$

is equal to $2.7 \times 10^{6} \mathrm{erg} \mathrm{cm}^{-2} \mathrm{~s}^{-1}$. The second term on the right hand side of Eq. (5) corresponds to the conductive flux along magnetic field lines

$F_{\text {cond, }}=\kappa \frac{\mathrm{d} T}{\mathrm{~d} h}$

where $\kappa=1.1 \times 10^{-6} T^{5 / 2} \mathrm{erg} \mathrm{s}^{-1} \mathrm{~cm}^{-1} \mathrm{~K}^{-1}$ is the Spitzer thermal conductivity and $\frac{\mathrm{d} T}{\mathrm{~d} h}$ is the absolute value of the temperature gradient. Accelerating the material upwards (downwards) by reconnecting field lines, means that there must actually be a cut-off from the region below (above) and thus the plasma will not lose energy by conduction along the field towards the reconnection site. In the directions away from the reconnection site, the temperature gradient and thus the conductive flux is small (cf. Fig. 4). This can also be justified by some simple calculations. Equating the relation above to $2\left(1.1 \times 10^{-6} T^{7 / 2} / L / 2\right)$ then a conductive flux equal to $0.44 \mathrm{erg} \mathrm{cm}^{-2} \mathrm{~s}^{-1}$ is obtained. From this estimate it can be concluded that the conductive flux is very small and can be neglected. It should be noted that both fluxes given above are upper limits, since we assume that the entire volume radiates or conducts the energy.

From the other two fluxes the kinetic energy flux can be deposited as heat, e.g. by forming shocks and then dissipating via viscosity and conduction. The enthalpy flux, as suggested by Pneuman and Kopp (1977), may contribute to maintain the coronal energy budget by balancing transition region emission when the gas flows back so that conduction from the coronal energy budget is no longer needed for this purpose. Adopting for the duration of the outflow+downflow phase of each event a time $t_{i}$ equal to $150 \mathrm{~s}$, the contribution of all mottles to the global energy flux can be found from the total individual energy flux multiplied by the area of each event $S_{i}$, the lifetime of the event and the birthrate divided by the surface of the $\operatorname{Sun} S_{\mathrm{o}}$, e.g.

$F_{\text {global, } \mathrm{t}}=\frac{F_{i, t} S_{i} t_{i} b}{2 S_{\mathrm{o}}}$

where the factor $1 / 2$ comes from the assumption that only half of them show the signature of reconnection each time. In Table 2 we summarize the contribution of each structure to each energy flux component, as well as the contribution of all events to the global energetics. Half of their sum gives the contribution of mottles to the energy budget of the solar corona (equal amounts go to the energy budget of the chromosphere). This flux is $\sim 20 \%$ of the total flux needed to maintain the solar corona. It should be noted that this value is overestimated since a part of the kinetic and enthalpy fluxes is converted into potential energy while the gas moves upward and only the remaining part is available for maintaining the coronal energy budget. The energy flux $F_{\text {total,t }}$ has to balance the total magnetic energy content $F_{\text {mag,t }}$ released at the reconnection site. Equating these two fluxes we can obtain a new estimate of the magnetic field. The mean magnetic field obtained by this way is of the order of $11 \mathrm{G}$.
Table 2. Energy fluxes.

\begin{tabular}{lcc}
\hline \hline $\begin{array}{l}\text { Flux in } \\
\mathrm{erg} \mathrm{cm}^{-2} \mathrm{~s}^{-1}\end{array}$ & Per event & Global \\
\hline$F_{\text {rad }}$ & $2.7 \times 10^{6}$ & $3.4 \times 10^{4}$ \\
$F_{\text {cond }}$ & 0.44 & $5.7 \times 10^{-3}$ \\
$F_{\text {kin }}$ & $3.4 \times 10^{6}$ & $4.4 \times 10^{4}$ \\
$F_{\text {enth }}$ & $3 \times 10^{6}$ & $3.9 \times 10^{4}$ \\
\hline$F_{\text {total }}$ & $9.1 \times 10^{6}$ & $1.2 \times 10^{5}$ \\
\hline$F_{\text {down }}$ & $7.5 \times 10^{6}$ & $9.7 \times 10^{4}$ \\
\hline
\end{tabular}

We should also mention that some external forces, like wave pressure forces and magnetic curvature forces, although we are not able to treat them quantitatively, might play an important role during the reconnection phase. Reconnection is thought to generate a lot of waves (e.g. the high frequency Alfvén waves necessary to explain the different kinetic temperatures of various atomic species in the open corona), and the Lorentz force due to the curvature of the magnetic field not only in, but also significantly above the reconnection site, might help to maintain Alfvén speed when the flow is about to decelerate due to conversion of kinetic into potential energy.

Downflow phase After the upward+downward phase downward motion follows. A mechanism has been proposed by Athay \& Holzer (1982) for the heating supplied to the chromosphere by the downflowing spicular material. This mechanism is based on the inference that spicules are raised well above the height that would be achieved by a projectile of the same initial velocity thereby obtaining gravitational potential energy much in excess of its initial kinetic energy. When the spicular material falls back to the chromosphere the excess gravitational energy will be converted to flow energy and to internal energy as the fall is slowed thus providing a source of heat for the atmosphere. If we assume that all the potential energy is converted to internal energy with no radiative losses then the energy flux supplied to the chromosphere during the return flow can be estimated. Assuming a mean length equal to $10 \mathrm{Mm}$, a mean inclination angle equal to $29^{\circ}$, and a downflowing flow speed, $v_{\mathrm{f}}$, equal to $11 \mathrm{~km} \mathrm{~s}^{-1}$ then from

$F_{\text {down }}=\frac{1}{2} \rho g L \cos \theta v_{\mathrm{f}}$

we find that the energy flux released per event is of the order of $2.9 \times 10^{6} \mathrm{erg} \mathrm{cm}^{-2} \mathrm{~s}^{-1}$. The factor of $1 / 2 \mathrm{in} \mathrm{Eq.} \mathrm{(12)} \mathrm{results}$ from the fact that not all the mass falls from the very top of the structure. The global flux released is obtained from

$F_{\text {down }, \mathrm{t}}=\frac{F_{\text {down }, i} S_{i} t_{i} b}{2 S_{\mathrm{o}}}$

and is equal to $3.7 \times 10^{4} \mathrm{erg} \mathrm{cm}^{-2} \mathrm{~s}^{-1}$. To this value the downward flux obtained during the reconnection phase must be added resulting in an energy flux of $7.5 \times 10^{6} \mathrm{erg} \mathrm{cm}^{-2} \mathrm{~s}^{-1}$ per event and a global flux of $9.7 \times 10^{4} \mathrm{erg} \mathrm{cm}^{-2} \mathrm{~s}^{-1}$. Thus the energy released by gravitationally accelerated downward falling 

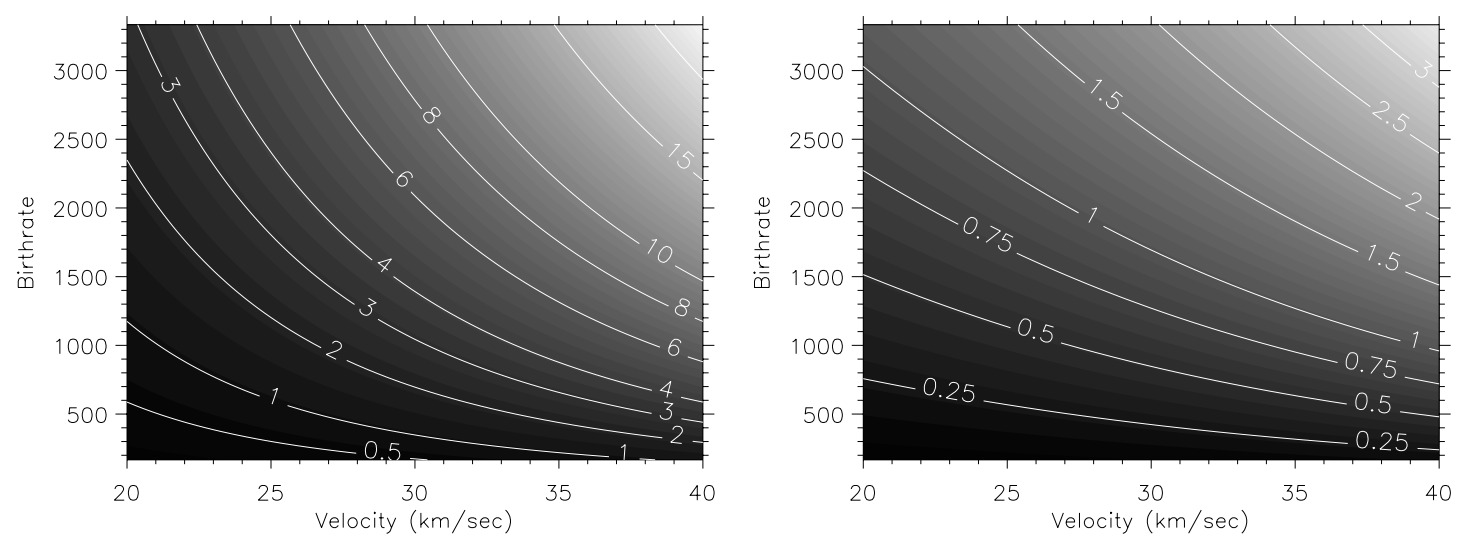

Fig. 6. Contour plot of the kinetic flux in units of $10^{4} \mathrm{erg} \mathrm{cm}^{-2} \mathrm{~s}^{-1}$ (left) and of the total magnetic energy flux in units of $10^{5} \mathrm{erg} \mathrm{cm}^{-2} \mathrm{~s}^{-1}($ right) that goes towards the corona as a function of axial velocity and mottle birthrate.

material is an insignificant factor in the global energy balance of the upper chromosphere (it can account for less than $\sim 2 \%$ of the chromospheric heating requirements).

Of course, the values of the different parameters, especially those of the axial velocity and of the birthrate may play an important role on the above estimated flux values. In Fig. 6 we give a contour plot of the kinetic energy flux that goes into heat (left) and of the total magnetic energy flux (right) for velocities in the range $20-40 \mathrm{~km} \mathrm{~s}^{-1}$ and total number of mottles found on the Sun in the range $5 \times 10^{4}-10^{6}$. It is obvious that for low velocities and low total number of mottles the contribution of these structures to the total magnetic energy and to the heating (even if we assume that all the kinetic flux goes into heating) of the solar corona is negligible. However, it is very interesting to note that as the velocity and the number of mottles increase the energy fluxes increase, as well as the percentage of the energy flux that goes into heating.

\section{Discussion}

The understanding of the dynamics of the chromospheric fine structures, like e.g. mottles/spicules, is an essential ingredient for the modelling of the solar atmosphere. There are strong indications that mottles are driven by magnetic reconnection, a process that can lead not only to energy release with implications for coronal heating, but also to the transfer of cool gas from the chromosphere to the corona. In this context, the ill-understood processes of heat and mass transfer from the chromosphere to the corona and vice-versa can be clarified by studying details of the dynamical behaviour of individual chromospheric structures. The deduced physical parameters of mottles and their temporal variations have been proved very useful in obtaining, at least, order of magnitude estimates of the different processes that contribute to the mass balance and heating of the solar chromosphere and corona and to derive estimates of the energy released by magnetic reconnection.

We have found that mottles supply material to the solar corona much in excess of that needed to compensate for coronal mass losses in the solar wind. The surplus material undoubtedly has to return to the chromosphere. Thus mottles are the physical means by which the overall mass balance in the solar atmosphere is established. Mottles' material returning to the surface provides an explanation for the considerable downflows observed in several transition region spectral lines at the network boundaries where these structures occur. Since it is already suggested that these downflows represent spicular material falling back to the chromosphere (Pneuman \& Kopp 1978; Withbroe 1983), this finding provides one more indirect evidence to the identity of these structures.

Mottles, as well as spicules (see Athay \& Holzer 1982), transport energy high up in the upper chromosphere and corona. Using some typical values for the velocity and the total number taken equal to that of spicules then the sum of the bulk kinetic energy, of the enthalpy energy and of the radiation losses could account for at most $\sim 20 \%$ of the total coronal energy losses. However, from all these energy fluxes only part of the kinetic energy is deposited as heat. If this is the case then mottles by themselves do not transfer enough energy to heat the corona. This could lead us to the following suggestions:

1. it is possible that the total energy released that goes into heat may be substantially larger from the kinetic energy (actually the part of the kinetic energy that is not converted to potential energy). The reason is that the reconnection can release a large fraction of its energy as MHD waves which travel far from the reconnection site. These may provide an energy that goes to the heat of the solar corona (Antiochos, private communication). Furthermore, we are regarding the visible mottle as resulting from the eruption of a small amount of magnetic flux that has become detached from below by reconnection. However, the eruption of magnetic flux into the corona, which is already permeated by magnetic field, will lead to current sheets at the interface between the old field and the new field. If the magnetic field in the corona contains currents, it necessarily contains free energy which may be released by reconnection. These two processes could provide the means by which the corona outside active regions is heated;

2. maybe the corona is in fact heated by a wide variety of mechanisms that each can provide a percentage of the required heating rate. In that case, the relative contributions of the various phenomena have to be determined quantitatively. The sheer complexity of the solar atmosphere 
suggests that it is not impossible that there are more than one mechanisms at work, although this possibility is unattractive for those looking for only one mechanism which could provide the total heating rate.

Moreover, we found that if we assume that even if all the gravitational potential energy gained during the mottles' rise is converted into internal energy as the material is flowing down, then the globally released energy by mottles can account for less than $\sim 2 \%$ of the chromospheric heating requirements.

There are, of course, large uncertainties in the reported flux estimates. One of them is the birthrate of these events, which is related to their number on the solar surface at each given moment. This number depends on the spatial resolution, instrument and line of observation and of several other factors. The other is the axial velocity. Using different velocities and total numbers values we are able to show that for a range of values mottles can significantly contribute to the solar atmosphere heating requirements. It is worth noting, e.g. that if we assume that we have the maximum mottle birthrate and maximum velocity then the total flux that goes towards the corona is comparable to the total coronal energy losses.

Clearly speaking, mottles could contribute to the coronal energy budget although they cannot be considered to be a significant heating source (unless the role of the different type of waves generated by the reconnection could be treated quantitatively and proven to be significant). In this context further work is needed which by combining the observational characteristics of the magnetic field related to these structures, their relationship with other small-scale transient events, e.g. blinkers, explosive events etc., their total number on the solar surface, and the derivation of accurate physical parameters by using nonLTE models will help to better establish their role in the heating of the solar atmosphere.

Acknowledgements. The THEMIS telescope is operated on the island of Tenerife by CNRS-CNR in the Spanish Observatorio del Teide of the Instituto de Astrofísica de Canarias. We would like to thank L. Fletcher, C. Mandrini, S. Antiochos and A. Anastasiadis for useful discussions and the referee R. Hammer for providing very detailed and helpful comments. K.T.'s research is funded by the European Commission through the European Solar Magnetism Network (contract HPRN-CT-2002-00313).

\section{References}

Alissandrakis, C. E., Tsiropoula, G., \& Mein, P. 1990, A\&A, 230, 200 Athay, R. G., \& Holzer, T. E. 1982, ApJ, 255, 743

Beckers, J. M. 1963, ApJ, 138, 648

Beckers, J. M. 1964, Ph.D. Thesis, Utrecht

Beckers, J. M. 1972, ARA\&A, 10, 73

Blake, M. L., \& Sturrock, P. A. 1985 ApJ, 290, 359

Bray, R. J., \& Loughhead, R. E. 1974, The Solar Chromosphere (London: Chapman and Hall)

Cargill, P. J. 1994, in Solar System Plasmas in Space and Time, AGU Monograph 84, ed. J. L. Burch, \& J. H. Waite Jr., 21

Forbes, T. G., \& Priest, E. R. 1987, Rev. Geophys., 25, 1583

Hansteen, V. H., Betta, R., \& Carlsson, M. 2000, A\&A, 360, 742

Matsuno, K., \& Hirayama, T. 1988, Sol. Phys., 117, 21

Heristchi, D., \& Mouradian, Z. 1992, Sol. Phys., 142, 21

Nagai, F. 1980, Sol. Phys., 68, 351

Pneuman, G. W., \& Kopp, R. A. 1977, A\&A, 55, 30

Pneuman, G. W., \& Kopp, R. A. 1978, Sol. Phys., 57, 49

Priest, E. R., \& Forbes, T. G. 2000, in Magnetic Reconnection, MHD Theory and Applications (Cambridge University Press)

Schrijver, C. J., Title, A. M., Van Ballegooijen, A. A., Hagenaar, H. J., \& Shine, R. A. 1997, ApJ, 487, 424

Sterling, A. C. 2000, Sol. Phys., 196, 79

Tsiropoula, G., Alissandrakis, C. E., \& Schmieder, B. 1993, A\&A, 271,574

Tsiropoula, G., \& Schmieder, B. 1997, A\&A, 324, 1183

Tziotziou, K., Tsiropoula, G., \& Mein, P. 2003, A\&A, 402, 361

Tziotziou, K., Tsiropoula, G., \& Mein, P. 2004, A\&A, 423, 1133

Ulmschneider, P. 1971, A\&A, 12, 297

Wang, H., Tang, F., Zirin, H., \& Wang, J. 1996, Sol. Phys., 165, 223

Withbroe, G. L., \& Noyes, R. W. 1977, ARA\&A, 15, 363

Withbroe, G. L. 1983, ApJ, 267, 825

Winebarger, A. R., Emslie, A. G., Mariska, J. T., \& Warren, H. P. 1999, ApJ, 526, 471 